1 Supplementary Information

2 Gaseous elemental mercury (GEM) fluxes over canopy of two typical

3 subtropical forests in south China

4 Qian $\mathrm{Yu}^{1}$, Yao $\mathrm{Luo}^{1}$, Shuxiao Wang ${ }^{1,2}$, Zhiqi Wang ${ }^{1}$, Jiming Hao ${ }^{1,2}$, Lei Duan ${ }^{1,2}$

5 'State Key Laboratory of Environmental Simulation and Pollution Control, School of Environment, Tsinghua University, 6 Beijing 100084, China.

$7 \quad{ }^{2}$ Collaborative Innovation Centre for Regional Environmental Quality, Tsinghua University, Beijing 100084, China.

8 Correspondence to: Lei Duan (lduan@tsinghua.edu.cn)

91 Calculation steps of turbulent transfer coefficient $(K)$ : Page S1

10 References Page S2

11 Table S1 Page S2

12 Table S2. Page S3

13 Figure $\mathrm{S} 1$. Page S4

14 Figure $\mathrm{S} 2$. Page S5

15 Figure S3.

Page S6

16 


\section{$18 \mathbf{1}$ Calculation steps of turbulent transfer coefficient $(\boldsymbol{K})$ :}

19 Turbulent transfer coefficient $K$ was calculated according to the similarity theory after the measurement of the wind speed and

20 temperature profile (Yu and Sun, 2006)

21 (1) Calculation of Richard Sunds $\left(R_{\mathrm{i}}\right)$ :

$22 \quad R_{i}=\frac{\mathrm{g}\left(\frac{T_{2}-T_{1}}{z_{2}-z_{1}}+\gamma_{\mathrm{d}}\right)}{T_{0}\left(\frac{u_{2}-u_{1}}{z_{2}-z_{1}}\right)^{2}}$,

23 where $g$ is the acceleration of gravity $\left(9.8 \mathrm{~m} \mathrm{~s}^{-2}\right), \gamma_{\mathrm{d}}$ is the dry adiabatic lapse rate $\left(0.00976 \mathrm{~K} \mathrm{~m}^{-1}\right), z_{1}$ and $z_{2}$ are the heights $(\mathrm{m})$,

$24 T_{1}, T_{2}$ and $T_{0}$ are the temperatures at two heights and the mean value $(\mathrm{K}), u_{1}$ and $u_{2}$ are wind speeds at two heights $\left(\mathrm{m} \mathrm{s}^{-1}\right)$.

25 (2) Determination of the initial atmospheric stability $\left(\zeta_{0}\right)$ :

$26 \zeta_{0}=\left\{\begin{array}{c}R_{i}, R_{i}<0 \\ \frac{R_{i}}{1-5 R_{i}}, 0 \leq R_{i} \leq 0.1, \\ 0.2, R_{i}>0.1\end{array}\right.$

27 (3) Determination of universal dimensionless gradient function $(\Phi(\zeta))$ :

$28 \Phi_{m}\left(\zeta_{0}\right)=\left\{\begin{array}{c}\left(1-15 \zeta_{0}\right)^{-1 / 4}, \quad \zeta_{0} \leq 0 \\ 1+5 \zeta_{0}, \quad \zeta_{0}>0\end{array}\right.$,

$29 \Phi_{h}=\Phi_{c}=\left\{\begin{array}{l}\Phi_{m}^{2}, \zeta_{0} \leq 0 \\ \Phi_{m}, \zeta_{0}>0\end{array}\right.$,

30 where $\Phi_{h}, \Phi_{c}$ and $\Phi_{m}$ are the universal functions of sensible heat, mercury, and momentum.

31 (4) Calculation of scales of speed $\left(u_{*}\right)$ and temperature $\left(\theta_{*}\right)$ :

$32 u_{*}=\frac{\kappa\left(u_{2}-u_{1}\right)}{\Phi_{m}\left(\zeta_{0}\right) \ln \left[\left(z_{2}-d\right) /\left(z_{1}-d\right)\right]}$,

$33 \theta_{*}=\frac{\kappa\left(T_{2}-T_{1}\right)}{\Phi_{h}\left(\zeta_{0}\right) \ln \left[\left(z_{2}-d\right) /\left(z_{1}-d\right)\right]}$,

34 where $d$ is the displacement of zero plane (m), equal to 0.7 times of the vegetation height, $\kappa$ is the Karman constant (0.4).

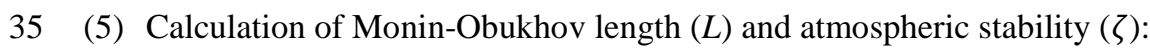

$36 L=\frac{u_{*}^{2}}{\kappa^{2} \frac{g}{\theta_{0}} \theta_{*}}$,

$37 \zeta=z / L$,

38 where $\theta_{0}=T_{0}$, and $z$ indicates the height related to the flux (m).

39 (6) If there is a large difference between $\zeta$ and $\zeta_{0}$, set $\zeta_{0}=\zeta$ and repeat steps (3)-(5) until $\zeta$ converging to one value. 
40 (7) Calculation of turbulent transfer coefficient $(K)$ using the parameters

$41 K=\frac{u_{*} K z}{\Phi_{c}(\zeta)}$,

\section{References}

43 Yu, G., and Sun, X.: The principle and method of terrestrial ecosystems flux observations. Higher Education Press, Beijing, 44 2006. (In Chinese)

45

46 Table S1. Sensors used for measuring meteorological parameters

\begin{tabular}{|c|c|c|c|}
\hline Sensor type & Version & Detection range & Accuracy \\
\hline Air temperature (two heights) & 43347-L (R. M. Young Inc., USA) & $-50 \sim 50^{\circ} \mathrm{C}$ & $\pm 0.1^{\circ} \mathrm{C}$ \\
\hline \multirow{4}{*}{$\begin{array}{c}\text { Wind speed and direction (two } \\
\text { heights) }\end{array}$} & \multirow{4}{*}{ WindSonic (Gill Inc., UK) } & Wind speed: $0 \sim 60$ & \\
\hline & & $\mathrm{m} / \mathrm{s}$ & $\pm 2 \%$ speed \\
\hline & & Wind direction: $0-$ & $\pm 3^{\circ}$ direction \\
\hline & & $359^{\circ}$ & \\
\hline \multirow{3}{*}{ Air temperature and humidity } & \multirow{3}{*}{ HMP 155A (Vaisala Inc., Finland) } & Temperature: -80- & \\
\hline & & $60^{\circ} \mathrm{C}$ & $\pm 0.2^{\circ} \mathrm{C}$ \\
\hline & & Humidity: $0.8-100 \%$ & \\
\hline Solar radiation & $\begin{array}{c}\text { CS300 (Campbell Scientific Inc., } \\
\text { USA) }\end{array}$ & $300-1120 \mathrm{~nm}$ & $\pm 5 \%$ \\
\hline \multirow{2}{*}{ Soil temperature and moisture } & \multirow{2}{*}{$\begin{array}{c}\text { CS } 616 \text { (Campbell Scientific Inc., } \\
\text { USA) }\end{array}$} & $\begin{array}{l}\text { Temperature: -30- } \\
\quad 70^{\circ} \mathrm{C}\end{array}$ & Temperature: $\pm 0.5^{\circ} \mathrm{C}$ \\
\hline & & Moisture: $0.05-0.5$ & Mo1sture: $\pm 5 \%$ \\
\hline \multirow{2}{*}{ Precipitation } & 52202/52203 (R. M. Young Inc., & \multirow{2}{*}{$\geq 0.1 \mathrm{~mm}$} & $2 \%(\leq 25 \mathrm{~mm} / \mathrm{h})$ \\
\hline & USA) & & $3 \%(\leq 50 \mathrm{~mm} / \mathrm{h})$ \\
\hline
\end{tabular}

47

48 
50 Table S2. Seasonal atmospheric GEM flux and meteorological parameters at QYZ and HT sites. Data format: mean value (variance), min 51 value $\sim$ max value.

\begin{tabular}{|c|c|c|c|c|c|c|c|}
\hline & & $\begin{array}{c}\text { Atmospheric GEM } \\
\text { concentration }\left(\mathrm{ng} \mathrm{m}^{-3}\right)\end{array}$ & $\begin{array}{l}\text { Air } \\
\text { temperature } \\
\left({ }^{\circ} \mathrm{C}\right)\end{array}$ & $\begin{array}{l}\text { Air humidity } \\
(\%)\end{array}$ & $\begin{array}{l}\text { Soil } \\
\text { temperature } \\
\left({ }^{\circ} \mathbf{C}\right)\end{array}$ & $\begin{array}{l}\text { Soil } \\
\text { moisture } \\
(\%)\end{array}$ & $\begin{array}{l}\text { Solar } \\
\text { radiation (W } \\
\left.\mathbf{m}^{-2}\right)\end{array}$ \\
\hline \multirow{4}{*}{ Winter } & \multirow{2}{*}{ QYZ } & $4.05(1.53)$ & $7.64(5.67)$ & $72.5(21.7)$ & $8.52(2.37)$ & $0.30(0.02)$ & $221(221)$ \\
\hline & & $1.64 \sim 11.7$ & $-4.51 \sim 25.9$ & $16.4 \sim 98.7$ & $3.90 \sim 15.2$ & $0.27 \sim 0.36$ & $0 \sim 846$ \\
\hline & \multirow{2}{*}{ HT } & $5.94(3.20)$ & $6.42(5.12)$ & $77.9(20.2)$ & $7.33(2.40)$ & $0.28(0.01)$ & $169(188)$ \\
\hline & & $1.32 \sim 22.9$ & $-5.15 \sim 24.0$ & $15.8 \sim 100$ & $1.78 \sim 14.3$ & $0.26 \sim 0.32$ & $0 \sim 857$ \\
\hline \multirow{4}{*}{ Spring } & \multirow{2}{*}{ QYZ } & $3.47(1.81)$ & $19.0(6.08)$ & $82.2(15.9)$ & $18.0(4.35)$ & $0.37(0.02)$ & $224(246)$ \\
\hline & & $0.97 \sim 17.4$ & $4.60 \sim 33.6$ & $31.3 \sim 98.4$ & $8.00 \sim 26.2$ & $0.31 \sim 0.47$ & $0 \sim 987$ \\
\hline & \multirow{2}{*}{ HT } & $5.50(2.91)$ & $16.7(5.56)$ & $86.4(14.2)$ & $16.1(3.99)$ & $0.28(0.02)$ & $201(232)$ \\
\hline & & $1.45 \sim 22.9$ & $4.93 \sim 32.1$ & $25.4 \sim 99.5$ & $7.42 \sim 25.1$ & $0.24 \sim 0.34$ & $0 \sim 971$ \\
\hline \multirow{4}{*}{ Summer } & \multirow{2}{*}{ QYZ } & $3.30(1.23)$ & $27.3(3.77)$ & $80.0(15.8)$ & $26.1(1.52)$ & $0.37(0.04)$ & $325(291)$ \\
\hline & & $1.60 \sim 8.83$ & $20.1 \sim 36.8$ & $35.9 \sim 98.3$ & $22.8 \sim 29.5$ & $0.28 \sim 0.52$ & $0 \sim 1000$ \\
\hline & \multirow{2}{*}{ HT } & $5.51(3.09)$ & $25.2(3.73)$ & $87.4(14.0)$ & $26.6(1.93)$ & $0.25(0.02)$ & $207(261)$ \\
\hline & & $1.43 \sim 21.4$ & $18.0 \sim 36.0$ & $41.4 \sim 99.7$ & $22.4 \sim 30.1$ & $0.21 \sim 0.31$ & $0 \sim 988$ \\
\hline \multirow{4}{*}{ Fall } & \multirow[b]{2}{*}{ QYZ } & $3.75(1.18)$ & $20.7(6.16)$ & $80.3(17.0)$ & $20.5(3.93)$ & $0.26(0.03)$ & $252(235)$ \\
\hline & & $1.42 \sim 8.76$ & $5.66 \sim 36.3$ & $32.4 \sim 100$ & $12.1 \sim 28.3$ & $0.22 \sim 0.35$ & $0 \sim 943$ \\
\hline & \multirow[b]{2}{*}{ HT } & $6.64(3.26)$ & $19.3(6.04)$ & $83.9(16.3)$ & $20.5(4.83)$ & $0.26(0.02)$ & $217(245)$ \\
\hline & & $156 \sim 229$ & $145 \sim 344$ & $346 \sim 100$ & $861 \sim 285$ & $023 \sim 031$ & $0 \sim 965$ \\
\hline
\end{tabular}




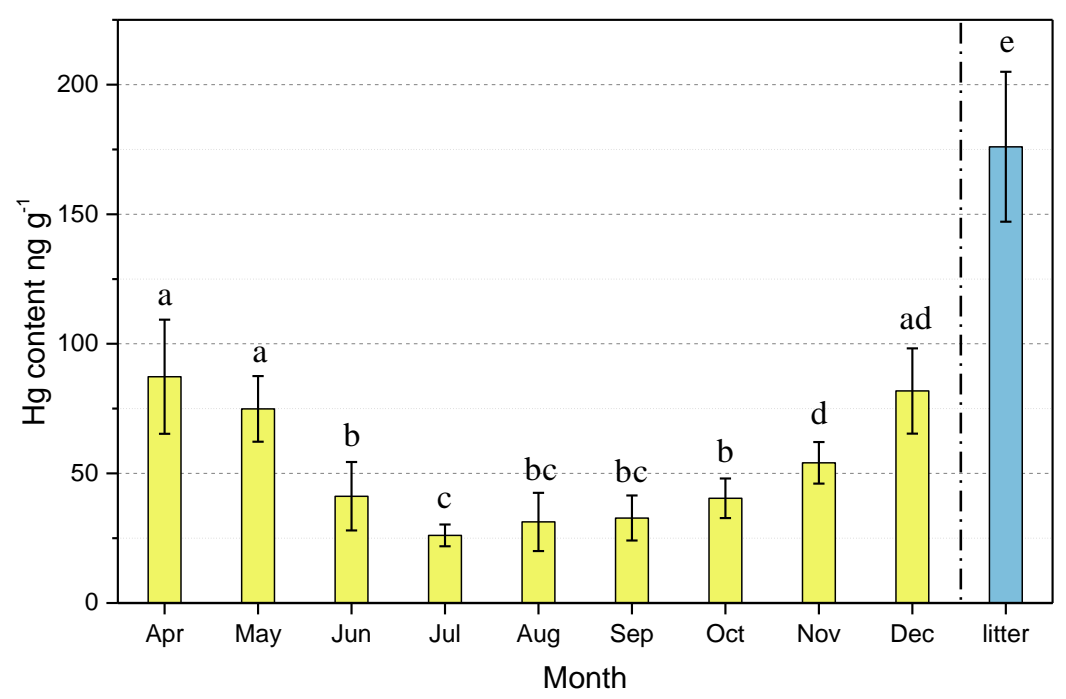

52

53 Figure S1. The monthly variation of $\mathrm{Hg}$ content of current-year foliage of cunninghamia lanceolata and the $\mathrm{Hg}$ content of litter at HT site.

54 Different letters in a column mean significant difference $(n=18, p<0.05)$.

55 


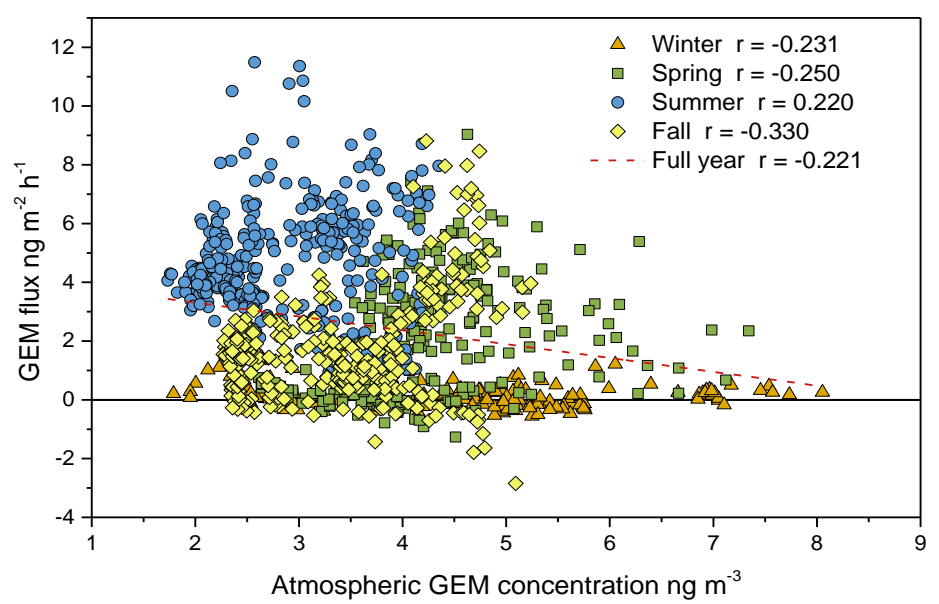

58 Figure S2. The correlation of GEM flux and atmospheric GEM concentration of soil at QYZ site (unpublished data). 

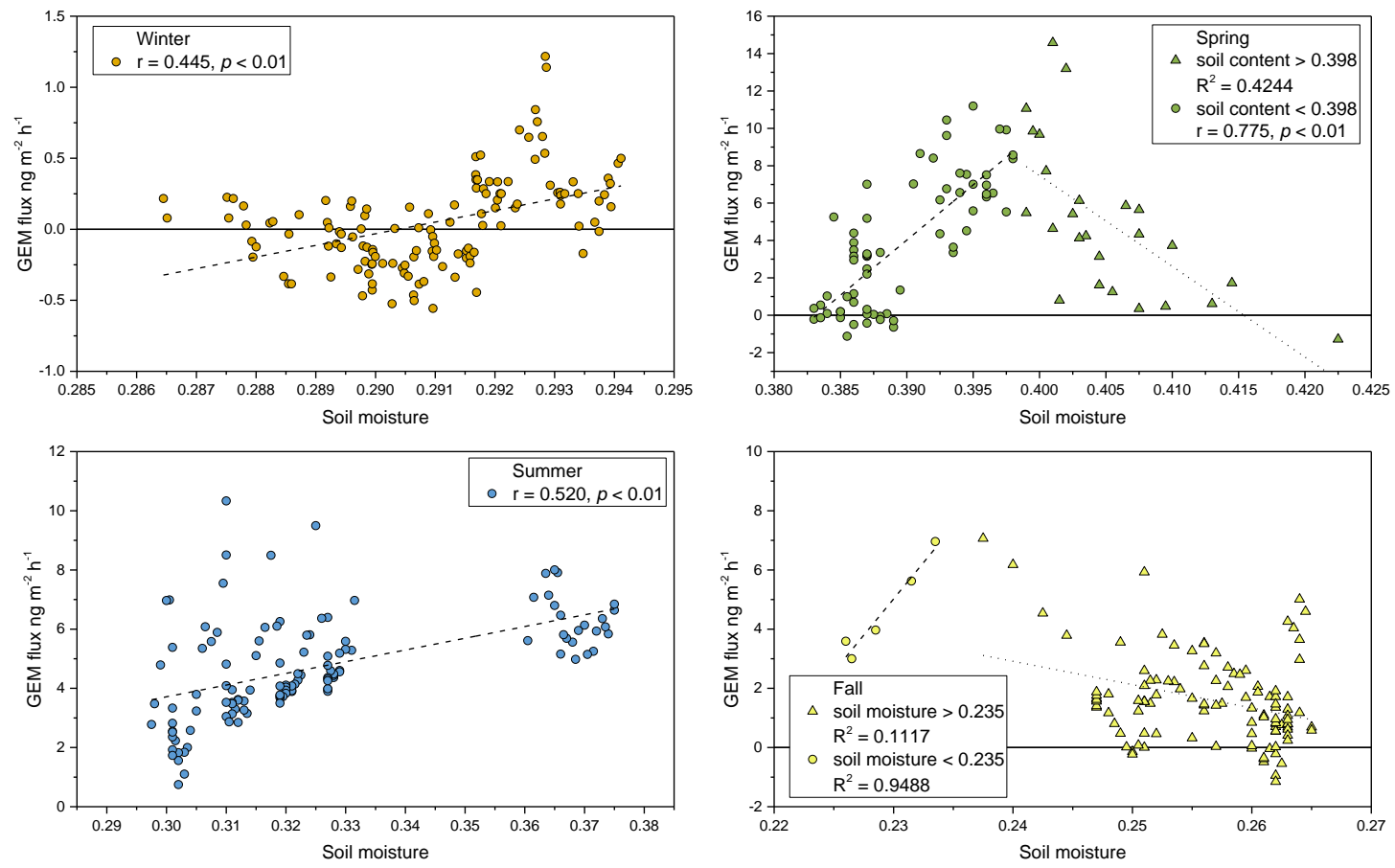

63 Figure S3. The soil GEM flux varied with the change of soil moisture in winter (a), spring (b), summer (c) and fall (d) at QYZ site 64 (unpublished data). 